\title{
Starburst Feedback in Local, Massive Galaxies
}

\author{
Crystal L. Martin $\dagger$ \\ Department of Physics, University of California, Santa Barbara, CA 93106, USA \\ email: cmartin@physics.ucsb.edu
}

\section{Outflows from Major-Mergers in the Local Universe}

Major mergers of gas-rich galaxies, each comparable in mass to the Milky Way, are rare at the present epoch. These events were readily identifed, however, two decades ago in far-infrared sky surveys (Soifer et al. 1986, 1987). Removal of the dust enshrouding these starbursts was almost immediately proposed as an evolutionary path to quasar formation (Sanders 1988). Recent measurements of the stellar velocity dispersion, rotation speed, and stellar surface brightness profile of these mergers suggest ULIRGs are indeed progenitors of field elliptical galaxies (Genzel et al. 2001; Tacconi et al. 2002).

Numerical simulations of galaxy mergers suggest feedback from starbursts and accreting (supermassive) black holes is quite important for understanding this transformation of spiral disks into field ellipticals via the ULIRG phase. Springel et al. (2005) demonstrate that strong AGN feedback, triggered by tidally induced inflow toward a supermassive black hole, could plausibly turn off the starburst activity. The spectacular pictures, see Figure 11 of Springel et al. for example, certainly leave the impression that the AGN wind devastates the galaxy. Should we conclude that AGN feedback is the magic elixir that separates the red and the blue galaxy populations?

I would argue that the burden of proof still lies with the observations. The macroscopic addition of the gas physics to these simulations is subject to many uncertainties. The simulations represent a giant leap forward on their own, but a robust solution is likely to require some empirical normalization. A close comparison with data has not yet been carried out, but there exists an abundance of new data.

The gas kinematics of many local ULIRG's have recently been studied using echellete spectra taken with the Keck II telescope (Rupke et al. 2002; Martin 2005; Rupke et al. 2005a,b,c,d; Martin 2006). Outflows were detected via Doppler-shifted, interstellar absorption lines. Since the background light source is the starburst in this case, there is no ambiguity between inflow and outflow. The equivalent width and depth of the absorption troughs constrain the column density of the ion, $\mathrm{NaI}$ for these studies, and the fraction of the continuum source covered by cool, outflowing gas. Outflows were discovered in nearly all ULIRGs, yielding typical speeds of $330 \pm 100 \mathrm{~km} \mathrm{~s}^{-1}$. The inferred mass flux in this cool phase of the outflow is sensitive to assumed ionization corrections and optical depth limits, but values of a few to ten percent the star formation rate seem likely.

Surprisingly, however, only the outflows in Seyfert 1's (see Rupke et al. 2005d) are clearly driven by supermassive black holes rather than starbursts. Many ULIRGs show evidence for highly obscured AGN (Seyfert 2 or LINER nuclei), yet the outflows appear to be driven predominately by starbursts rather than accreting black holes. First, the infrared colors of many of these galaxies are cool, indicating the far-infrared photons are emitted from a fairly large region. Second, the absorption velocities are far below the

\section{$\dagger$ Packard Fellow}


escape velocity from the broad line region. Third, and perhaps most revealing, is that the outflows emanate from a region much larger than the galactic nucleus.

This large spatial extent is illustrated for a typical low-redshift ULIRG, IRAS172080014, in Figure 1. The interstellar absorption kinematics are mapped across the entire luminous galaxy, and the outflow is found to cover it. The measured rotation of this outflow is of particular note because the velocity gradient matches the underlying rotation curve (modulo a large, constant offset - i.e. the outfow). Angular momentum conservation can be used to argue that the outflowing gas is not far above the disk(s), leading to the conclusion that the outflow is launched from a large area - not just the nucleus. The estimated outflow energy, momentum, and mass loss rate are also consistent with a supernova-driven outflow based on the measured star formation rates.

It remains possible that these ULIRGs have simply not reached their quasar phase. Springel et al. (2005) find the starburst and AGN (active galactic nucleus) activity are slightly offset in time, so that the remnant is seen in different stages as the merger progresses. In principle, this evolution can be followed observationally by estimating the progression of the merger from the separation of the nuclei and the prominence of tidal tails (Murphy et al. 2001). Due to various ambiguities and limited sample sizes, the wind surveys are not in complete agreement about the results. Rupke et al. (2005b) find no dependence of the outflow properties on the merger state, but Martin (2006) claims the outflows become weaker in the single nuclei systems.

\section{Conclusion}

Should one be concerned that local ULIRGs do not clearly show the type of outflows required to permanently turn off star formation? Recent observational results do raise some concerns but are far from definitive. In addition to the timing issue described above, two other issues need further investigation. First, the measured amount of mass loss in ULIRG outflows depends critically on ionization corrections for the cool phase (Murray et al. 2006) and more data for the hot phase. Second, the wind acceleration mechanism is still debated - energy-driven or momentum-driven - radiation pressure or ram pressure. It therefore remains unclear exactly how far the cool outflow may travel into the halo from a dynamical standpoint. Indeed, dwarf galaxies appear to be the source of many warm, metal-enriched regions in the local IGM (see references in Martin 2006b). The feedback from quasars is likely much stronger at higher redshift, especially near peak quasar activity, i.e. around $2<z<3$.

\section{Acknowledgements}

C. L. M. is supported by the David and Lucile Packard Foundation.

\section{References}

Genzel, R. et al. 2001, ApJ, 563, 527.

Martin, C. L. 1999, ApJ, 513, 156.

Martin, C. L. 2004, in Carnegie Observatories Astrophysics Series, Vol. 4: Origin and Evolution of the Elements, ed. A. McWilliam and M. Rauch (Pasadena: Carnegie Observatories).

Martin, C. L. 2005, ApJ, 621, 227 (Paper I).

Martin, C. L. 2006, ApJ, 647, 222.

Martin, C. L. 2006b, Astrophysics Update 2, J W Mason (Editor), ISBN: 3-540-30312-

X Springer Berlin Heidelberg New York, C Praxis Publishing Ltd, Chichester, UK 2006 (also on astro-ph). 

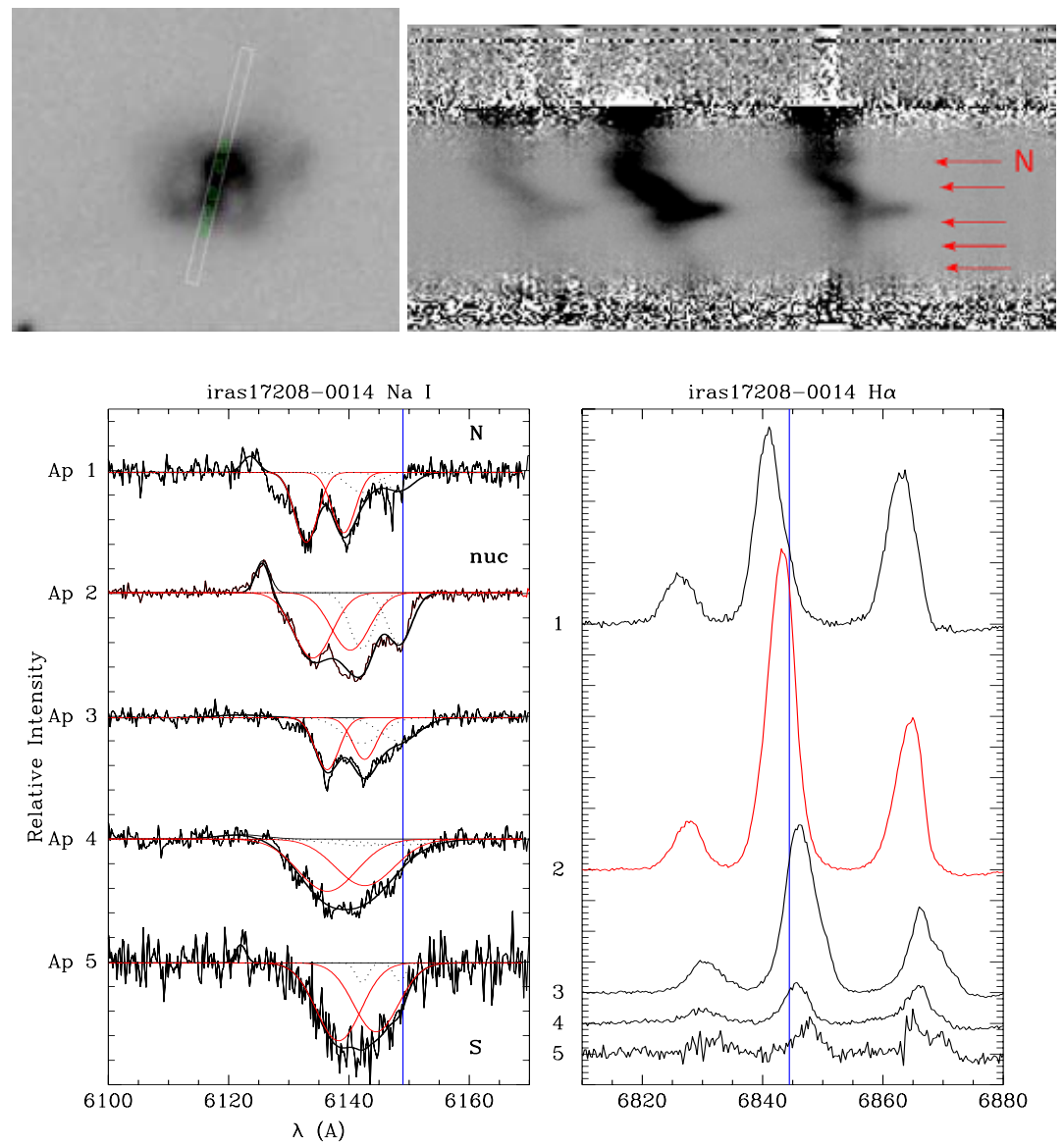

Figure 1. Figure 1 of Martin (2006) for IRAS17208-0014. (Top Left) Position of the apertures on an R band image from Murphy et al. (1996). North is up, and east is to the left. The slit is $20^{\prime \prime}$ long. (Top Right) The $\mathrm{H} \alpha$ plus [NII] $\lambda \lambda 6548,6584$ emission lines along the slit; wavelength increases to the right. Spectral apertures and slit orientation are indicated. (Bottom Right) Extracted $\mathrm{H} \alpha$ spectra with apertures and slit orientation marked. (Bottom Left) Extracted spectra at $\mathrm{Na} I \lambda \lambda 5890,96$. The nuclear spectrum (nuc), aperture number, and slit orientation are marked. The vertical line indicates the systemic velocity of the $\lambda 5896$ line. Two Na I doublets are plotted ; the velocity of one is fixed at the systemic velocity (dotted), and the velocity of the dynamic component is fitted (solid). The Na I absorption is highly blueshifted across both of the merging galaxies; the outflow speed is $400 \mathrm{~km} \mathrm{~s}^{-1}$ at the nucleus. The $\mathrm{H} \alpha$ emission reveals rotation across the main galaxy and the prograde nature of the encounter. The $\mathrm{Na}$ I absorption shows the same velocity gradient as the $\mathrm{H} \alpha$ emission here, but the velocities are offset such that the Doppler shift is negative across the entire galaxy.

Murphy, T. W., Jr., Soifer, B. T., Matthews, K., \& Armus, L. 2001, ApJ, 559, 201.

Murphy, T. W., Jr. et al. 1996, AJ, 111, 1025.

Murray, N. et al. 2006, astro-ph/0609213.

Rupke, D. S., Veilleux, S., \& Sanders, D. B. 2002, ApJ, 570, 588.

Rupke, D. S., Veilleux, S., \& Sanders, D. B. 2005a, ApJS, 160, 87.

Rupke, D. S., Veilleux, S., \& Sanders, D. B. 2005b, ApJS, 160, 115.

Rupke, D. S., Veilleux, S., \& Sanders, D. B. 2005c, ApJ, 631, L37.

Rupke, D., Veilleux, S., \& Sanders, D. B. 2005d, ApJ, 632, 751. 
Sanders, D. B. 1988, ApJ, 325, 74.

Soifer, B. T. et al. 1986, ApJ, 303, L41.

Soifer, B. T. et al. 1987, ApJ, 320, 238.

Springel, V. et al. 2005, MNRAS, 361, 776.

Tacconi, L. J. et al. 2002, ApJ, 580, 73.

\section{Discussion}

Mo: Given the results you presented here, are theorists still allowed to make the assumption that the mass outflow rate is comparable to the star formation rate in a starburst galaxy?

MARTIN: You need to specify whether you mean winds that escape from the halo potential or simply outflows from the disk, which may turn around at large distances in the halo. The interpretation presented in Martin $(1999,2004)$ is that the bulk of the hot wind can escape from a dwarf galaxy (rotation speed less than $130 \mathrm{~km} \mathrm{~s}^{-1}$ ). This conclusion is based on emission-measure weighted X-ray temperatures and HI rotation curves of about a dozen starburst galaxies. The gas mass in the coronal phase (from X-ray measurements) is of the same order of magnitude as the gas mass in the warm-ionized phase (determined from $\mathrm{H} \alpha$ measurements), and this mass flux is shown to be a few times the star formation rate. Hence, you can say that hot gas leaves low-mass, starburst galaxies at rates up to a few times the star formation rate. The fate of the warm-ionized gas in dwarfs is model dependent, but this is only a factor of two correction (roughly). The hot gas that we detect directly in massive galaxies is not hot enough to escape the halo potential, but the observations are not sensitive to the hottest phase of these winds. Our inadequate knowledge of the degree of mixing between the hot wind and cooler, entrained gas leaves a great deal of uncertainty about whether this undetected, hotter gas contains significant mass. My presentation here focused on the cool gas in outflows from more massive galaxies, which we can easily detect in UV/optical spectra. The mass flux in this phase is less, a few tens of percent of the star formation rate. Preliminary analysis of $\mathrm{X}$-ray observations of a few of these objects indicate that they have hot winds that reach at least $30 \mathrm{kpc}$. So, in the local universe, yes in dwarf starbursts and not necessarily for more massive galaxies.

Cid Fernandes: Could you clarify whether outflows in Seyfert 1's are AGN dominated but not in Seyfert 2's? And, if so, does that pose a problem for unified models?

MARTIN: No, this is not necessarily a problem for unified models. I think an accurate description of the Rupke et al. (2005d) work would be that the outflows from Seyfert 1's usually present a fast component that is unlikely associated with a starburst wind. Since the Seyfert 1 outflows appear to have smaller opening angles (than starburst winds), the AGN wind may not intersect the sightline toward Seyfert 2's. Although a prominent starburst wind is not required in all Seyfert 1's, I believe the limits on the maximal starburst wind would show large variations from object to object. I'll have to refer you back to the Rupke et al. data for a more precise answer. 